\title{
R14 - IDENTIFICAÇÃO DE ANTÍGENOS IMUNODOMINANTES EM PACIENTES COM LEPTOSPIROSE ATRAVÉS DE MICROARRANJO DE PROTEÍNAS
}

Carolina Lessa-Aquino $^{1 *}$, Jozelyn Pablo $^{2}$, Li Liang ${ }^{2}$, Elsio A. Wunder Jr. ${ }^{3}$, Cristiane Pinheiro Pestana ${ }^{1}$, Guilherme S. Ribeiro ${ }^{4,5}$, Ricardo Galler ${ }^{1}$, Mitermayer G. Reis ${ }^{4}$, Albert I. $\mathrm{Ko}^{3}$, Philip L. Felgner ${ }^{2}$, Marco Alberto Medeiros ${ }^{1}$.

${ }^{1}$ Bio-Manguinhos, Fundação Oswaldo Cruz, RJ, Brasil. ${ }^{2}$ Departmento de Medicina, Divisão de Doenças Infecciosas, Universidade da Califórnia Irvine, Irvine, CA, USA. ${ }^{3}$ Departamento de Epidemiologia de Doenças Microbianas, Universidade de Yale, New Haven, CT, USA. ${ }^{4}$ Centro de Pesquisa Gonçalo Moniz, Fundação Oswaldo Cruz, Salvador, BA, Brasil, ${ }^{5}$ Instituto de Saúde Coletiva, Universidade Federal da Bahia, Salvador, BA, Brasil.

INTRODUÇão: A tecnologia de microarranjo de proteínas é uma potente ferramenta de alto desempenho para identificar alvos candidatos a vacinas e marcadores diagnósticos para doenças infecciosas. Existe, hoje, uma ausência de medidas profiláticas e de testes diagnósticos eficazes para leptospirose, doença zoonótica causada por bactérias do gênero Leptospira sp.

OBJETIVO: Identificar novos alvos protéicos que possam ser empregados como candidatos à vacina e/ou marcadores diagnósticos para leptospirose, utilizando a plataforma de microarranjo de proteínas.

Metodologia: O microarranjo foi construído compreendendo 2241 proteínas de Leptospira interrogans sorovar Copenhageni e investigou-se a resposta por anticorpos IgG de 274 indivíduos, sendo 80 pacientes em fase aguda da doença, 80 em fase convalescente e 114 indivíduos saudáveis provenientes de área com transmissão endêmica e não endêmica para doença.

RESUltados: Foram encontrados 16 antígenos capazes de identificar casos agudos de leptospirose e 18 capazes identificar casos convalescentes da doença. Antígenos como LipL32, Loa22, GroEL e os domínios das proteínas Lig foram previamente descritos 
como sendo reconhecidos por soros de pacientes humanos, atuando como prova de conceito para a plataforma de microarranjo protéico. Novos antígenos também foram identificados no estudo, como a proteína hipotética LIC10215, que mostrou alta acurácia na identificação de casos agudos e convalescentes de leptospirose.

CONCLUSÃo: Os antígenos imunodominantes identificados demonstram potencial uso no desenvolvimento de vacinas e de novos ensaios diagnósticos, assim como no melhoramento dos testes disponíveis no mercado. Estudos complementares estão em andamento para avaliar o desempenho diagnóstico desses antígenos nos formatos de ELISA e/ou de teste rápido. 